\title{
Evaluation of Quantitative Real-Time PCR and Platelia Galactomannan Assays for Diagnosis of Disseminated Talaromyces marneffei Infection
}

\section{Xinlei $\mathrm{Li}^{1,3 \dagger}$, Yanqing Zheng ${ }^{1,2 \dagger}$, Fengyao $\mathrm{Wu}^{4 \dagger}$, Dongdong $\mathrm{Mo}^{1 \dagger}$, Hao Liang ${ }^{2,5}$, Gang Liang ${ }^{6}$, Rufan Yan ${ }^{1}$, Jazeer Abdul Khader ${ }^{1}$, Nianning $\mathrm{Wu}^{4^{*}}$, Cunwei Cao ${ }^{1,2^{*}}$ \\ 1.Department of Dermatology and Venereology, the First Affiliated Hospital of Guangxi Medical University. 2.Guangxi Key Laboratory of AIDS Prevention and Treatment, Guangxi Medical University. 3.Department of Mycology, Yulin Hospital of Dermatology. 4.The Fourth People's Hospital of Nanning. 5.School of Public Health and Life sciences Institute, Guangxi Medical University. 6.School of Preclinical Medicine of Guangxi Medical University}

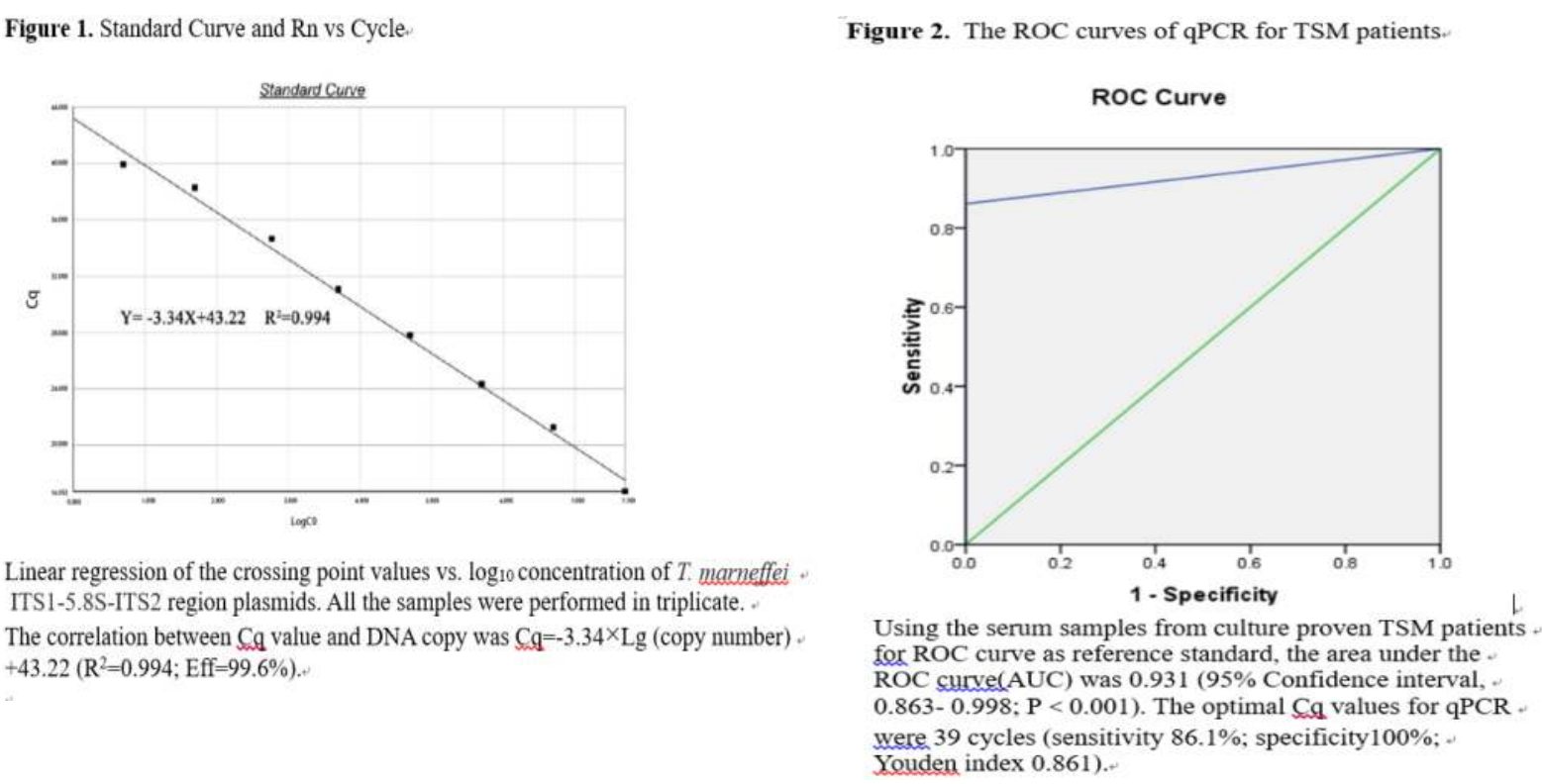

Background: Talaromyces (Penicillium) marneffei is an emerging pathogen that causes significant morbidity and mortality in immunocompromised patients in endemic regions, such as Southeast Asia. This study compared the diagnostic performance of a well-validated realtime quantitative PCR (qPCR) assay targeting the ITS1-5.8S-ITS2 region and a Platelia galactomannan (GM) assay for the diagnosis of talaromycosis marneffei (TSM) using serum samples from patients with or without HIV.

Methods: Establish a TaqMan qPCR to detect and identify T. marneffei DNA coding for ITS1-5.8S-ITS2 region. Thirty-six patients with TSM were enrolled for laboratory diagnosis using qPCR and GM assays. Serum samples were first tested by qPCR and later for the GM antigen using an ELISA assay. qPCR was carried out using an automated quantitative fluorescence PCR cycler, and the results were analyzed using a receiver operating characteristic curve. Sensitivity and specificity of serum qPCR assay were calculated. GM levels were determined using Platelia Aspergillus enzyme immuno-assay. The GM optical density cutoff index was $\geq 0.5$. The sensitivities of both diagnostic methods were compared using Fisher's test.

Results: A total of 36 patients were enrolled in this study. The mean patient age was 38.64 years (range 1-69 years). Fourteen (38.89\%) patients had HIV co-infection, while the other $22(61.11 \%)$ had no detectable underlying diseases. The qPCR system proved to be highly specific for T. marneffei with an amplification product of $202 \mathrm{bp}$ (primers and probe show in Table 1). The correlation between Cq value and DNA copy was $\mathrm{Cq}=-3.34 \times \mathrm{Lg}$ (copy number)+43.22 $\left(\mathrm{R}^{2}=0.994\right.$; $\mathrm{Eff}=99.6 \%$ ) (Figure 1). Using serum samples from cultureproven TSM patients for the ROC curve as a reference standard, the AUC was 0.931 (95\% confidence interval, 0.863-0.998; $\mathrm{p}<0.001$ ). The optimal $\mathrm{Cq}$ value for qPCR was 39 cycles (sensitivity $86.1 \%$; specificity $100 \%$; Youden index 0.861) (Figure 2).

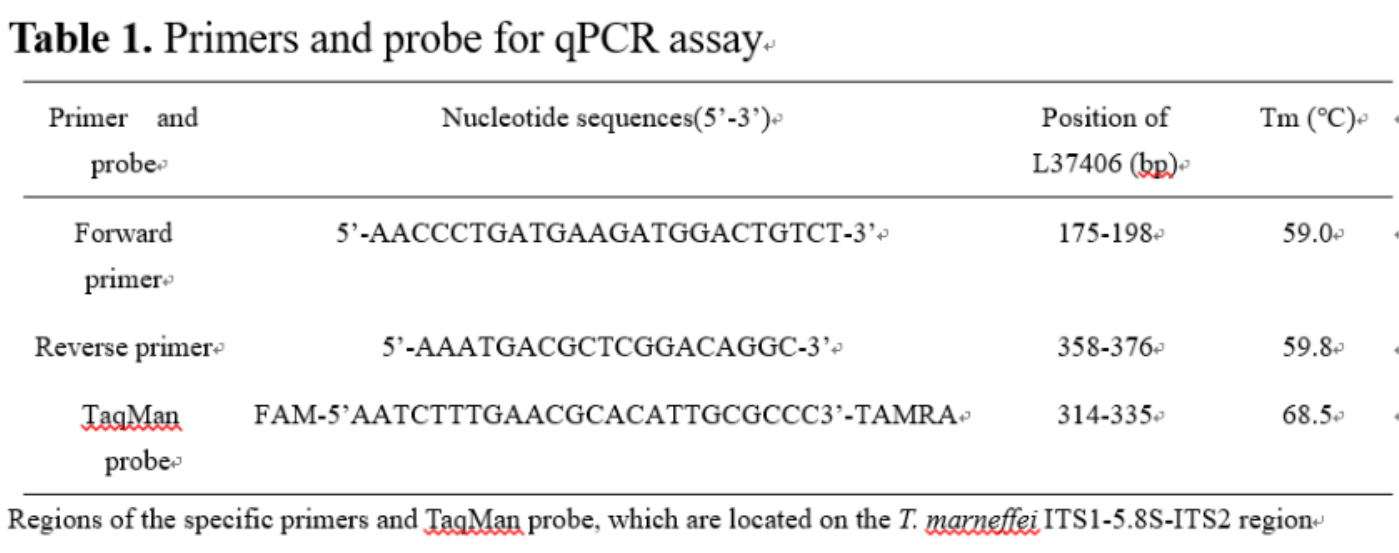

The results showed that this novel qPCR method is highly sensitive and specific for detection of T. marneffei DNA in serum samples; the limit of detection and species specificity were five copies of DNA and $100 \%$, respectively. In assaying serum samples from 36 patients with TSM, the sensitivity of the qPCR method was $86.11 \%$ (31/36), including $20 / 20(100 \%)$ patients with fungemia and $11 / 16(68.75 \%)$ without fungemia. For GM assay, the sensitivity was $80.56 \%$ (29/36) when the GM optical density cutoff index was $\geq 0.5$, including $19 / 20$ (95\%) patients with and 10/16 (62.5\%) without fungemia.

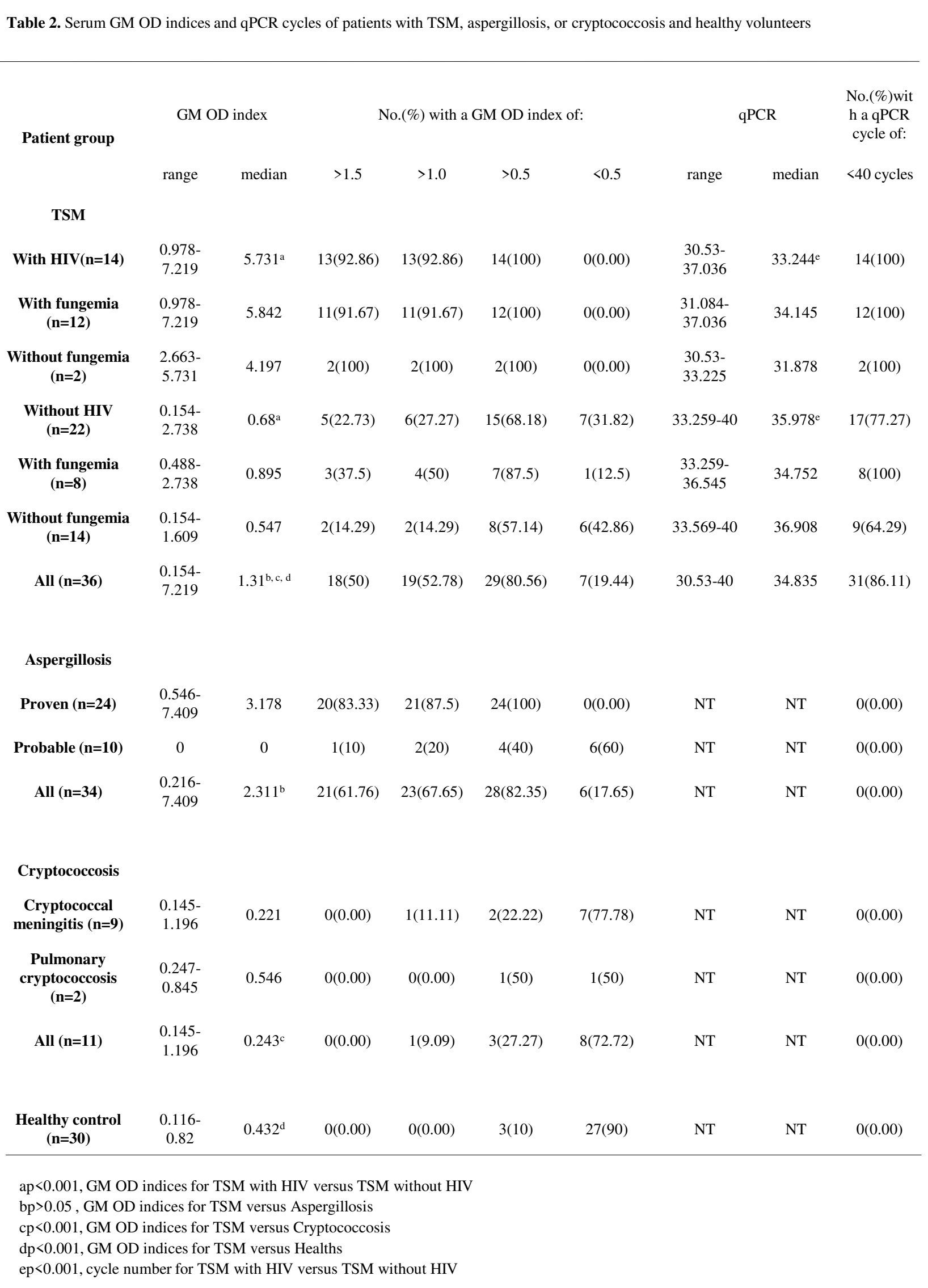

Conclusion: These results indicate that a novel qPCR assay and GM assay can be valuable tools in the diagnosis of T. marneffei infection. Serum is a convenient hematological sample source for T. marneffei DNA quantification. Combining GM and qPCR assays is a more accurate and appropriate method for diagnosis of T. marneffei infection in endemic areas. 